\title{
Empirical Studies on Feature Selection for Software Fault Prediction
}

\author{
Jiaqiang Chen ${ }^{1}$, Shulong Liu ${ }^{1}$, Xiang Chen ${ }^{1,2}$, Qing Gu ${ }^{1}{ }^{*}$, Daoxu Chen ${ }^{1}$ \\ ${ }^{1}$ State Key Laboratory for Novel Software Technology \\ Nanjing University, Nanjing, China \\ chensonglin1989@gmail.com \\ ${ }^{2}$ School of Computer Science and Technology \\ Nantong University, Nantong, China \\ xchencs@ntu.edu.cn
}

\begin{abstract}
Classification based software fault prediction methods aim to classify the modules into either fault-prone or non-faultprone. Feature selection is a preprocess step used to improve the data quality. However most of previous research mainly focus on feature relevance analysis, there is little work focusing on feature redundancy analysis. Therefore we propose a two-stage framework for feature selection to solve this issue. In particular, during the feature relevance phase, we adopt three different relevance measures to obtain the relevant feature subset. Then during the feature redundancy analysis phase, we use a cluster-based method to eliminate redundant features. To verify the effectiveness of our proposed framework, we choose typical real-world software projects, including Eclipse projects and NASA software project KC1. Final empirical result shows the effectiveness of our proposed framework.
\end{abstract}

\section{Categories and Subject Descriptors}

D.2.8 [Software Engineering]: Metrics-complexity measures, performance measures

\section{General Terms}

Reliability, Experimentation

\section{Keywords}

Software Fault Prediction, Feature Selection, Relevance Analysis, Redundancy Analysis

\section{INTRODUCTION}

Software fault prediction (SFP) can allocate limited resource in a cost-effective manner by predicting fault-proneness of software modules. Classification is a popular approach used for SFP and the main task is to categorize modules,

\footnotetext{
*Correspondence author Qing Gu, E-mail: guq@nju.edu.cn.
}

Permission to make digital or hard copies of all or part of this work for personal or classroom use is granted without fee provided that copies are not made or distributed for profit or commercial advantage and that copies bear this notice and the full citation on the first page. To copy otherwise, to republish, to post on servers or to redistribute to lists, requires prior specific permission and/or a fee.

Internetware '13, October 23-24, 2013, Changsha, China

Copyright 2013 ACM 978-1-4503-2369-7/13/10 ...\$15.00. represented by a set of software metrics, into fault-prone (FP) or non-fault-prone (NFP). Until now, many classification based SFP methods have been proposed [1, 2, 3]. However the effectiveness of these methods depends on the data quality represented as a set of software metrics. Therefore selecting a proper subset of metrics becomes an integral component of the model building process and here we mainly focus on this issue in this paper.

Feature selection is a preprocessing step to build classifiers used for SFP. It can be viewed as the process of identifying and removing as many irrelevant and redundant features as possible. In SFP, previous research mainly focused on searching for relevant features. However redundant features can also affect the effectiveness of SFP. Therefore we propose a two-stage framework used for feature selection. In our framework, we combine feature relevance analysis and redundancy analysis to remove both irrelevant features and redundant features. In particular, during the feature relevance analysis stage, we consider three different relevance measures to obtain the relevant feature subset. Then during the feature redundancy analysis stage, we use a clusteringbased method to remove redundant features.

To investigate the effect of our proposed framework, we designed and performed a set of empirical studies. We choose some real-world projects including Eclipse and $\mathrm{KC} 1$ and use AUC value and F-measure as metrics. In addition, we also use FCBF and CFS as our baseline methods. Final result shows that the methods considering redundancy analysis perform better than the methods only using relevance analysis and FCBF in most cases. However, we also note that the methods considering redundancy analysis has no obvious advantage when comparing with CFS.

The contribution of this paper can be highlighted as follows: (1) To the best of our knowledge, we firstly propose a novel two-stage approach for feature selection. (2) We designed and performed empirical studies on real-world projects to investigate the effectiveness of this framework.

\section{RELATED WORK}

In this section, we briefly summarize the related work and show the motivation of our approach.

Existing feature selection algorithms can be classified into two categories: the filter approach and the wrapper approach [4]. In the filter approaches, features are selected before the data mining algorithm is run. While in the wrapper approaches, they use the target data mining algorithm as a black box to find the best subset of attributes. Gao et al. applied some feature selection algorithms to SFP for a large 
legacy software system in telecommunications [5]. Their experimental results demonstrated that removing $85 \%$ of software metric features did not adversely affect results, and in some cases can even improved results. Wang et al. considered different ensembles of feature selection algorithms [6]. They indicated that ensembling a few algorithms is effective and sometimes can even better than ensembling many or all of the algorithms. Rodriguez et al. evaluated different filter approaches and wrapper approaches [7]. Final empirical result concluded that wrapper approaches were better than filter approaches but at the cost of a high computational cost. However after removing the irrelevant features, the remaining features are closely related to the classes and this inevitably leads to feature redundancy issue. In addition, redundant features can also affect the speed and accuracy of the trained classifiers.

In our empirical studies, we also adopt CFS [8] and FCBF [9] feature selection methods as our baseline methods. In particular, CFS method evaluates the worth of a subset of attributes by considering the individual predictive ability of each feature along with the degree of redundancy between them. In this method, subsets of features that are highly correlated with the class while having low inter correlation are preferred. FCBF method is based on the correlation measure in relevance and redundancy analysis and is used in conjunction with an attribute set evaluator.

\section{OUR TWO-STAGE FEATURE SELECTION FRAMEWORK}

In this paper, we propose a two-stage feature selection framework. This framework includes feature relevance analysis stage and redundancy analysis stage. Each stage can be implemented by different methods. In the first stage, we mainly measure the relevance of features by feature-class correlations. Here we use three different correlation measures which will be introduced in Section 3.1. In the second stage, we mainly measure the redundancy within a feature subset by inter-feature correlations. We apply a clusteringbased method, which will be introduced in Section 3.2, to eliminate redundancy features in the relevant feature subset. Therefore, this framework can be instanced into 6 different methods. In particular, three methods use both relevance analysis and redundancy analysis, and the other three methods only use relevance analysis.

\subsection{Relevance Analysis Stage}

Relevance analysis is used to select features most relevant to target class. Here we choose three different relevance measures, which is chi-square (CHI), symmetrical uncertainty (SU), and ReliefF (RLF), to weight the correlation between features and classes.

Chi-square is a nonparametric statistical measure used to examine the distribution of the class as it relates to the values of the given feature [10]. It can be computed by the following formula:

$$
C H I=\sum_{i=1}^{r} \sum_{j=1}^{n_{c}} \frac{\left(O_{i, j}-E_{i, j}\right)^{2}}{E_{i, j}}
$$

Here $r$ is the number of distinct values of the feature, $n_{c}$ is the number of classes (in SFP, $n_{c}$ is 2), $O_{i, j}$ is the observed number of instances with value $i$ in class $j$, and $E_{i, j}$ is the expected number of instances with value $i$ in class $j$

Symmetrical uncertainty measures entropy introduced by information theory and treats a pair of variables symmetrically [11]. It can be computed by the following formula:

$$
S U(X, Y)=2 \times \frac{I G(X \mid Y)}{H(X)+H(Y)}
$$

Here $H(X)$ is the entropy of a discrete random variable $X$ (i.e., the feature). Suppose $p(x)$ is the prior probabilities for all values of $x$, and $p(x \mid y)$ is the posterior probabilities of $x$ given the value of $y$ (i.e., the class), $H(X)$ can be computed by the formula $-\sum_{x \in X} p(x) \log p(x)$. The information gain $I G(X \mid Y)$ can measure the amount by which the entropy of $\mathrm{X}$ decreases and can be computed by the formula $H(X)-$ $\sum_{y \in Y} p(y) \sum_{x \in X} p(x \mid y) \log p(x \mid y)$. Note that for the data sets with continuous-valued features, the off-the-shelf MDL method was used to discretize the continuous values.

ReliefF is an instance based feature ranking technique, which can search for the nearest neighbors of instances from both the same and different classes, and can measure how well a feature differentiates instances from different classes. For a feature $X$, its RLF measure can be computed by the following formula:

$$
R L F(X)=\frac{\sum_{i=1}^{m}\left(\operatorname{diff}\left(x_{i}, x_{M}\right)-\operatorname{diff}\left(x_{i}, x_{H}\right)\right)}{m}
$$

Here $m$ is the number of randomly sampled instances and $x_{i}$ is the feature value of the $i$ th instance $O_{i}$. For $O_{i}$, both its nearest neighbor $O_{H}$ from the same class and the nearest neighbor $O_{M}$ from a different class are found. The the difference between the feature values (i.e., diff $\left(x_{i}, x_{M}\right)$ and $\left.\operatorname{diff}\left(x_{i}, x_{H}\right)\right)$ can be computed by the following formula:

$$
\operatorname{diff}\left(x_{1}, x_{2}\right)=\frac{\left|x_{1}-x_{2}\right|}{x_{\max }-x_{\min }}
$$

Here $x_{\max }$ and $x_{\min }$ denote the maximum and minimum values of $X$.

\subsection{Redundancy Analysis Stage}

However relevance analysis can only capture the relevance of features to target classes and cannot discover redundancy among features. Therefore we further conduct redundancy analysis to eliminate redundant features. Here we use a clustering-based method. The clustering-based method uses the similarity between features and we choose symmetrical uncertainty as the measure which is introduced in Section 3.1 .

This method groups features into clusters and then select$\mathrm{s}$ a representative feature for each cluster. To cluster these features, this method starts by calculating pairwise similarities between features, and then it constructs a $k$ nearest neighbor graph over the features. The method then selects the feature with the most compact neighborhood and removes all its neighbors. This process is repeated with the remaining features until all features are either selected or removed.

\section{EMPIRICAL STUDY}

In our empirical study, we want to answer the following two research questions. 
Table 1: The Summary of the Datasets

\begin{tabular}{|c|c|c|c|}
\hline Data Set & \#Metrics & \#Modules & \#Faulty Modules \\
\hline Eclipse 2.0 & 198 & 6729 & $2611(38.80 \%)$ \\
\hline Eclipse 2.1 & 198 & 7888 & $2139(27.12 \%)$ \\
\hline Eclipse 3.0 & 207 & 661 & $415(62.78 \%)$ \\
\hline KC1 & 94 & 145 & $60(41.38 \%)$ \\
\hline
\end{tabular}

RQ1: In our framework, can the methods using redundant analysis perform better than the methods only using relevance features?

RQ2: In our framework, can the methods using redundant analysis perform better than CFS and FCBF.

\subsection{Experimental Design}

To evaluate the effectiveness of our proposed method, we choose the Eclipse project and NASA software project KC1. These data can be downloaded from the PROMISE data repository ${ }^{1}$.

The eclipse data sets contain 198-207 features, including code complexity metrics and abstract syntax tree oriented metrics. To guarantee the generality of our results, we use the file-level data for Eclipse 2.0, 2.1 and use the packagelevel data for Eclipse 3.0. We also preprocess the original data by removing all nonnumeric features and converting the pre-release fault data into a binary class attribute: FP and NFP. The NASA project KC1 is a storage managemen$\mathrm{t}$ system for receiving and processing ground data written by Java programming language. This data set includes 145 instances with 95 features each. The summarization information is listed in Table 1.

In our proposed two-stage framework, we choose three different relevance measures in feature relevance analysis, and we use a cluster-based method in feature redundancy analysis. Then the framework can be instanced into 6 different methods. These methods are: (1) CHI, (2) SU, (3) RLF, (4) $\mathrm{CHI}+\mathrm{C}$, (5) $\mathrm{SU}+\mathrm{C}$, (6) RLF+C. Here CHI means in this method we use CHI metric to perform the relevance analysis and do not perform the redundancy analysis. $\mathrm{CHI}+\mathrm{C}$ meas in this method we use CHI metric to perform the relevance analysis and use the cluster-based method to perform the redundancy analysis. In addition, we also use FullSet, CFS, and FCBF as our baseline methods. Here FullSet methods means we choose all features. The detail of CFS and FCBF can be found in Section 2 .

During the classifier construction phase, we employ three different classification algorithms in our empirical studies. They are Naive Bayes (NB), C4.5, and IB1. Here NB is a probability-based algorithm, C4.5 is a decision tree learning algorithm, and IB1 is an instance-based learning algorith$\mathrm{m}$. The implementation of these algorithms is supported by Weka software package ${ }^{2}$.

In our empirical study, we choose two metrics F-measure and AUC to measure the effectiveness of different methods. F-measure represents a harmonic mean between recall and precision of a classifier. A receiver operating characteristic (ROC) curve is a graphical approach for displaying the tradeoff between true positive rate and false positive rate of a classifier. The area under the ROC curve (AUC) provides another approach for evaluating which classifier is better on

\footnotetext{
${ }^{1}$ https://code.google.com/p/promisedata

${ }^{2}$ http://www.cs.waikato.ac.nz/ml/weka/
}

average. The detail of F-measure and AUC can be found in [4].

\subsection{Result Analysis}

As there exist randomness in our approach, we perform 10 independent runs to get a high statistical confidence. In each run, we use 10-fold cross-validation method to compute the metric value of each method. The final mean value of F-measure and AUC value is shown in Table 2 and Table 3. In these tables, E20, E21, E30, and KC1 denote Eclipse 2.0, 2.1, 3.0, and NASA KC1 projects respectively. NB, C45, and IB1 denote Naive Bayes, C4.5, and IB1 classification algorithms respectively.

For RQ1, when considering F-measure value shown in Table 2, the methods using redundant analysis performs better than the methods only using relevance analysis for most of projects. In particular, when using IB1 as the classification algorithm, the methods using redundant analysis obviously performs better than the methods only using relevance analysis in all of four projects. When using NB as the classification algorithms, the methods using redundant analysis almost performs better except for two projects (i.e., Eclipse 3.0 and $\mathrm{KC} 1$ projects). When using $\mathrm{C} 4.5$ as the classification algorithm, the methods using redundant analysis performs even worse except for Eclipse 3.0 project. However these methods only lose $2 \%$ to $3 \%$.

When considering AUC value shown in Table 3, for most of projects, the methods using redundant analysis performs better than the methods only using relevance analysis. In particular, when using IB1 and NB as the classification algorithms, the methods using redundant analysis obviously performs better than the methods only using relevance analysis, except for $\mathrm{KC} 1$ project. When using IB1 as the classification algorithm in $\mathrm{KC} 1$, one of methods (i.e., $\mathrm{RLF}+\mathrm{C}$ ) performs little worse than those only using RLF to relevance analysis, while the other two of our method $\mathrm{CHI}+\mathrm{C}$ and $\mathrm{SU}+\mathrm{C}$ perform better.

For RQ2, when considering F-measure value shown in Table 2 , for eclipse 2.0 and 2.1 projects, the methods using redundant analysis performs better than FCBF except for $\mathrm{RLF}+\mathrm{C}$ in eclipse 2.1. when using $\mathrm{C} 4.5$ as the classification algorithm, the methods using redundant analysis performs little worse for eclipse 3.0 project. For KC1 project, the methods using redundant analysis performs better than FCBF when using IB1 as the classification algorithm. While using $\mathrm{C} 4.5$ as the classification algorithm, the methods using redundant analysis performs worse than FCBF.

When considering AUC value shown in Table 3, for eclipse $2.0,2.1$, and 3.0 projects, the methods using redundant analysis obviously perform better than FCBF. However, we also find that the methods using redundant analysis performs little worse on $\mathrm{KC} 1$ project. For $\mathrm{KC} 1$ project, the methods using redundant analysis loses 6 times in all 9 comparison$\mathrm{s}$, including $\mathrm{RLF}+\mathrm{C}, \mathrm{CHI}+\mathrm{C}$ in IB1 classification algorith$\mathrm{m}, \mathrm{RLf}+\mathrm{C}$ in $\mathrm{NB}$ classification algorithm, and all the three methods we proposed in C4.5 classification algorithm. During the comparison between the methods using redundant analysis and CFS, we find that these methods have the similar performance (i.e., no method always perform better the other methods.

\subsection{Threats to Validity}

In this subsection, we mainly discuss some potential threat- 
Table 2: The Mean Value of F-measure Value

\begin{tabular}{|c|c|c|c|c|c|c|c|c|c|c|c|c|}
\hline Method & $\mathrm{E} 20+\mathrm{NB}$ & $\mathrm{E} 21+\mathrm{NB}$ & $\mathrm{E} 30+\mathrm{NB}$ & $\mathrm{KC} 1+\mathrm{NB}$ & $\mathrm{E} 20+\mathrm{C} 45$ & $\mathrm{E} 21+\mathrm{C} 45$ & $\mathrm{E} 30+\mathrm{C} 45$ & $\mathrm{KC} 1+\mathrm{C} 45$ & $\mathrm{E} 20+\mathrm{IB} 1$ & $\overline{\mathrm{E} 21+\mathrm{IB} 1}$ & E30+IB1 & $\overline{\mathrm{KC} 1+\mathrm{IB} 1}$ \\
\hline FullSet & 0.3870 & 0.4321 & 0.5029 & 0.4775 & 0.5864 & 0.5243 & 0.7397 & 0.5798 & 0.6133 & 0.5468 & 0.7454 & 0.6151 \\
\hline CFS & 0.4539 & 0.4838 & 0.5159 & 0.5770 & 0.5819 & 0.5223 & 0.7317 & 0.6717 & 0.5883 & 0.5181 & 0.7204 & 0.6313 \\
\hline FCBF & 0.3734 & 0.3974 & 0.4904 & 0.4983 & 0.5167 & 0.4923 & 0.7543 & 0.7177 & 0.4356 & 0.4254 & 0.6831 & 0.5987 \\
\hline $\mathrm{CHI}$ & 0.3929 & 0.4683 & 0.4508 & 0.4858 & 0.5630 & 0.5043 & 0.7796 & 0.6305 & 0.5305 & 0.4503 & 0.7126 & 0.5675 \\
\hline SU & 0.4518 & 0.4429 & 0.4555 & 0.5081 & 0.4957 & 0.5048 & 0.7800 & 0.6709 & 0.5037 & 0.4685 & 0.7060 & 0.6153 \\
\hline RLF & 0.5322 & 0.2939 & 0.7263 & 0.6689 & 0.5032 & 0.0929 & 0.7593 & 0.4627 & 0.4998 & 0.4211 & 0.6802 & 0.5194 \\
\hline $\mathrm{CHI}+\mathrm{C}$ & 0.4357 & 0.4789 & 0.4724 & 0.4906 & 0.5454 & 0.5163 & 0.7584 & 0.6643 & 0.5593 & 0.5031 & 0.7132 & 0.6388 \\
\hline $\mathrm{SU}+\mathrm{C}$ & 0.4675 & 0.4838 & 0.4708 & 0.5060 & 0.5542 & 0.5142 & 0.7510 & 0.6784 & 0.5589 & 0.4878 & 0.7122 & 0.6570 \\
\hline $\mathrm{RLF}+\mathrm{C}$ & 0.5965 & 0.5192 & 0.6927 & 0.6325 & 0.5657 & 0.4714 & 0.7362 & 0.6595 & 0.5932 & 0.5037 & 0.7332 & 0.5889 \\
\hline
\end{tabular}

Table 3: The Mean Value of AUC Value

\begin{tabular}{|c|c|c|c|c|c|c|c|c|c|c|c|c|}
\hline Method & $\mathrm{E} 20+\mathrm{NB}$ & $\mathrm{E} 21+\mathrm{NB}$ & $\mathrm{E} 30+\mathrm{NB}$ & $\mathrm{KC} 1+\mathrm{NB}$ & $\mathrm{E} 20+\mathrm{C} 45$ & $\mathrm{E} 21+\mathrm{C} 45$ & E $30+\mathrm{C} 45$ & $\mathrm{KC} 1+\mathrm{C} 45$ & $\mathrm{E} 20+\mathrm{IB} 1$ & $\mathrm{E} 21+\mathrm{IB} 1$ & $\mathrm{E} 30+\mathrm{IB} 1$ & $\mathrm{KC} 1+\mathrm{IB} 1$ \\
\hline FullSet & 0.7055 & 0.7658 & 0.7114 & 0.8040 & 0.6598 & 0.6405 & 0.6588 & 0.7110 & 0.7038 & 0.7128 & 0.6260 & 0.7061 \\
\hline CFS & 0.7149 & 0.7677 & 0.7491 & 0.8127 & 0.6718 & 0.6709 & 0.6676 & 0.7232 & 0.6866 & 0.6966 & 0.6192 & 0.7026 \\
\hline FCBF & 0.6403 & 0.7217 & 0.6826 & 0.7851 & 0.6546 & 0.7244 & 0.6553 & 0.7438 & 0.6574 & 0.6971 & 0.5701 & 0.7249 \\
\hline CHI & 0.6694 & 0.7841 & 0.6517 & 0.7771 & 0.7125 & 0.7243 & 0.6682 & 0.6899 & 0.6496 & 0.6556 & 0.6173 & 0.6808 \\
\hline SU & 0.7079 & 0.7515 & 0.6533 & 0.8096 & 0.6744 & 0.7270 & 0.6657 & 0.7171 & 0.6203 & 0.6589 & 0.6087 & 0.6964 \\
\hline RLF & 0.6369 & 0.6725 & 0.6211 & 0.7468 & 0.6792 & 0.6174 & 0.6155 & 0.6175 & 0.6229 & 0.6345 & 0.5711 & 0.6714 \\
\hline $\mathrm{CHI}+\mathrm{C}$ & 0.7210 & 0.7786 & 0.6982 & 0.7992 & 0.6829 & 0.7240 & 0.6589 & 0.7055 & 0.6629 & 0.6874 & 0.6346 & 0.7154 \\
\hline $\mathrm{SU}+\mathrm{C}$ & 0.7166 & 0.7743 & 0.6958 & 0.8099 & 0.6984 & 0.7355 & 0.6557 & 0.7255 & 0.6648 & 0.6786 & 0.6264 & 0.7330 \\
\hline $\mathrm{RLF}+\mathrm{C}$ & 0.6860 & 0.7270 & 0.7170 & 0.7539 & 0.6992 & 0.7291 & 0.6608 & 0.7150 & 0.6887 & 0.6827 & 0.6388 & 0.6630 \\
\hline
\end{tabular}

$\mathrm{s}$ for our empirical studies. Threats to internal validity mainly come from the incorrect program implementation or corrupted data. To avoid these threats, we downloaded the data from the PROMISE data repository and thus the correctness is guaranteed. In addition, we also tested our programs with simple programs to detect the faults. Threats to external validity affect the result generalization. The first threat is the representativeness of our subjects. However, these subjects are commonly used by other researchers $[1,2,3]$. The second threat is from the choose of relevance analysis and redundancy analysis methods. However, the chosen methods are representative and in the future, we will adopt more methods to verify the conclusion in this paper.

\section{CONCLUSION AND FUTURE WORK}

In this paper, to improve the effectiveness of SFP, we propose a two-stage framework on feature selection. During the relevance analysis, we use three different correlation measures. Then during the redundancy analysis phase, we use a cluster-based method to eliminate feature redundancy. In empirical study, we choose real-world software projects, including Eclipse project and NASA software project KC1. Final result can guide software testers to perform better feature selection in SFP and then further reduce the cost of software development and development.

In the future, we want to consider the following potential issues. Firstly we want to adopt more relevance analysis and redundancy analysis methods and investigate the effect on feature selection. Secondly we want to apply our approach to more project data developed by other programming languages, such as $\mathrm{C}++, \mathrm{C} \#$, or Python. Last but not least we want to incorporate our proposed framework into software development in reality.

\section{Acknowledgments}

We would like to thank anonymous reviewers for their insightful and constructive comments. This work is supported in part by The NSFC Projects under Grant No. 61202006 and 61373012, the University Natural Science Research Project of Jiangsu Province under Grant No. 12KJB520014, The Open Project of State Key Laboratory for Novel Software Technology at Nanjing University under Grant No. KFKT2012B29, and the Nantong Application Research Plan under Grant No. BK2012023.

\section{REFERENCES}

[1] T. Menzies, J. Greenwald, and A. Frank, "Data mining static code attributes to learn defect predictors," IEEE Transactions on Software Engineering, vol. 33, no. 1, pp. 2-13, 2007.

[2] S. Lessmann, B. Baesens, C. Mues, and S. Pietsch, "Benchmarking classification models for software defect prediction: A proposed framework and novel findings," IEEE Transactions on Software Engineering, vol. 34, no. 4, pp. 485-496, 2008.

[3] Q. Song, Z. Jia, M. Shepperd, S. Ying, and J. Liu, "A general software defect-proneness prediction framework," IEEE Transactions on Software Engineering, vol. 37, no. 3, pp. 356-370, 2011.

[4] P.-N. Tan, M. Steinbach, and V. Kumar, Introduction to Data Mining. Addison-Wesley Longman Publishing Company, 2005.

[5] K. Gao, T. M. Khoshgoftaar, H. Wang, and N. Seliya, "Choosing software metrics for defect prediction: an investigation on feature selection techniques," Software Practice and Experience, vol. 41, no. 5, pp. 579-606, 2011.

[6] H. Wang, T. Khoshgoftaar, and A. Napolitano, "A comparative study of ensemble feature selection techniques for software defect prediction," in Proceedings of International Conference on Machine Learning and Applications, 2010, pp. 135-140.

[7] D. Rodriguez, R. Ruiz, J. Cuadrado-Gallego, and J. Aguilar-Ruiz, "Detecting fault modules applying feature selection to classifiers," in Proceedings of International Conference on Information Reuse and Integration, 2007, pp. 667-672.

[8] M. A. Hall, Correlation-based feature selection for machine learning. The University of Waikato, 1999.

[9] L. Yu and H. Liu, "Feature selection for high-dimensional data: A fast correlation-based filter solution," in Proceedings of the International Conferencce on Machine Learning, 2003, pp. 856-863.

[10] A. C. Cameron and P. K. Trivedi, Regression Analysis of Count Data, 2nd edition. Cambridge University Press, 1998.

[11] W. H. Press, B. P. Flannery, S. A. Teukolsky, and W. T. Vetterling, Numerical recipes in C. Cambridge University Press, 1988. 